\title{
XXXl.
}

\section{Ueber den Zoisit und seine Beziehung zum Epidot, so wie über die Zusammensetzung des letzteren.}

Von

Rammelsberg.

(Aus d. Bericht d. Berl, Akarlemie.)

Der Zoisi scheint anfänglich mit dem weissen Strahlstein oder Tremolith verwechselt worden zu sein. Im Anfang dieses Jahrhunderts fand sich auf der Saualpe in Kärnthen ein Mineral, verwachsen mit Quarz, Granat, Augit und Cyanit, welches nach dem Baron von $\mathrm{Z}$ ois den Namen erhielt, von $\mathrm{K}$ arsten beschrieben und von $\mathrm{Klap}$ roth untersucht wurde. Später ergab sich das Vorkommen des Zoisits auch am Fichtelgebirge u. s. w. Hauy vereinigte ihn mit seinem Epidol, oder dem Mineral, welches glasiger Strahlstein genannt, und nachher von Werner als Pistacit bezeichnet wurde. Die grosse Mehrzahl der Mineralogen ist bis in die neueste Zeit der Annahme H a u's gefolgt, und hat Zoisit, Pistacit und Manganepidot als Abänderungen des Epidots angesehen.

Indessen hat schon Steffen s $^{*}$ hervorgehoben, dass der Zoisit nicht blos durch die Farbe, sondern auch durch Glanz und Art der Spaltbarkeit sich rom Epidot unterscheide. Zwanzig Jahre später (1831) erfolgte eine Untersuchung von Brooke, woraus sich ergab, dass der Zoisit in der Krystallform und Spaltbarkeit vom Epidot verschieden ist ${ }^{* *}$.

Leider sind einigermassen deutliche und messbare Krystalle von Zoisit höchst selten. Ihr System ist allerdings das zwei und eingliedrige wie beim Epidot. Allein

*) Oryktognosie 1,74.

${ }^{* *}$ ) Phillips Mineralogy by Brooke and Miller. 
während letzterer in unsymmetrischen Prismen mit gerader Endfläche und symmetrischen Zuschärfungsflächen erscheint (ein gewendetes zwei- und eingliedriges System nach dem Ausdruck von Weiss), sind die Zoisitprismen symmetrisch. Sie sind gewöhnlich sechsseitig, vier- und zweiwinklig, so dass sie demnach als Combinationen eines rhombischen Prismas wit einer geraden Abstumpfungsfläche betrachtet werden müsseu. Der stumpfe Winkel dieses rhombischen Prismas beträgt nach Brooke $116^{\circ} 16^{\prime}$. Ich fand ihn an der Varietät aus der Fusch $115^{0} 45^{\prime}-116^{\circ} 0$. Die scharfe Kante ist gerade abgestumpft durch eine Fläche, welche mit den beiden Prismenflächen Winkel von $121^{\circ} 52^{\circ}$ (Brooke) bildet, die auch ich $=122^{\circ}$ gefunden habe. B rooke beschreibt auch das Vorkommen des zweifach stumpferen Prismas von $145^{\circ} 48$.

Sind nun schon die eben genannten Flächen der Horizontalzone selten deutlich, sondern meist nur durch starke Streifung angedeutet, so fehlen die Endflächen fast immer. Brooke führt indessen zwei Zuschärfungstächenpaare mit schieflaufender Kante an, und giebt die Neigung der stumpferen von ihnen zu dem stumpferen Prisma $=$ $123^{\circ} 30^{\prime}$.

Der 'Zoisit hat nur eine deutliche Spaltungsrichtung, der Abstumpfungsfläche der scharfen Kanten des Prismas entsprechend, weicht also auch hierdurch vom Epidot ab.

Miller vergleicht die Formen und Strukturverhältnisse des Zoisits mit denen des Euklases, und glaubt, beide seien isomorph.

Indessen scheint mir durch diese Beobachtungen der krystallographische Unterschied von Zoisit und Epidot noch nicht streng erwiesen.

Das symmetrisch sechsseitige Prisma des Zoisits kam beim Epidot nur in der Horizontaizone gesucht werden, und hier treffen wir $\mathrm{Hauy}$ 's Fläche $\mathrm{O}$ (der Weiss den Ausdruck - - 1/3 $\dot{a}: 1 / 8 b: c$ gegeben hat), welche nach Ersterem unter $121^{\circ} \mathbf{2 3}^{\prime}$ gegen die erste Spaltungsfläche $M$ geneigt ist; zwei Flächen $O$ müssen demnach über $P$ (der geraden Endfäche für das unsymmetrische Epidotprisma) unter $117^{\circ} 14^{\prime}$ geneigt sein. Diese beiden Winkel 
weichen von denen des Zoisits $122^{\circ}$ und $116^{\circ}$ nicht so sehr weit ab. Möglicherweise wäre beim Zoisit die Horizontalzone hauptsächlich entwickelt, während es beim Epidot die Vertikalzone ist.

Auch die Spaltbarkeit spricht nicht gegen die Identität der Formen, denn Brooke's (Miller's) Spaltungsfläche $b$ würde nach jener Annahme dem $M$ des Epidots entsprechen, dessen andere Spaltungsrichtung $\mathrm{T}$ in dem Zoisit vielleicht weniger deutlich ist.

Bei den Zweifeln, die dieser Gegenstand übrig lässt, hielt ich es für zweckmässig, zu untersuchen, ob dic chemische Zusammensel:ang eine Trennung beider Mineralien rechtfertige.

Klaproth's Analysen zufolge sind die Bestandtheile des Zoisits und des Epidots dieselben. Nur ergeben diese so wie spätere Versuche von Bucholz, Geffken u. A., dass der Zoisit etwas reicher an Kieselsäure ist, als der Epidot, und dass in ihm nur wenige Proc. Eisenoxyd sich finden, der Gehalt an Thonerde daher grösser ist. Bezüglich des allgemeinen chemischen Verhaltens zeigen beide deshalb viel Aehnlichkeit. An und für sich werden sie von Säuren nicht gut zersetzt. Wird aber Zoisit in Stücken oder pulverig zum Glühen erhitzt, , so wird er dann als feines Pulver von Säuren unter Gallertbildung leicht und vollkommen zerlegt. Nach $\mathrm{H}$ ermann geschicht dies zwar bei dem Epidot nur unvollkommen; doch habe ich schon vor längerer Zeit nachgewiessen, dass auch der geglühte Epidot von Säuren vollständig zersetzt wird.

Wir stellen nun die bekannt gewordenen Zoisitanalysen zusammen.

\section{Zoisit von der Saualpe.}

Er ist am zahlreichsten untersucht worden.

a. Grünlichgrauer krystallisirter $Z$.; spec. Gew. $=3,315$. Von Quarz, Cyanit, Augit und Granat begleitet. Klaproth.

b. Gelblichbrauner krystallisirter; spec. Gew. $=\mathbf{3 , 2 6 5}$. Mit Quarz verwachsen. Klaproth. 
c. Mürber Z. vom Radelgraben, röthlichweiss. Klap roth

d. Analyse von Thomson.

e. Analyse von Kulesza, unter Schrötter's Leitung.*

Kieselsäuro a. b. c. a d

Thonerde

Eisenoxyd

45

$47,5 \quad 44$

d.

e.

\section{Kalkerde}

$29 \quad 29,3 \quad 32$

29,49

44,00

Glühverlust

$\begin{array}{rrrrr}3 & 4,5 & 2,5 & 7,20 & 4,92 \\ 21 & 17,5 & 20 & 22,95 & 17,77\end{array}$

$$
\frac{-}{98}-\frac{0,75}{99,75} \quad \frac{1,36}{98,5} \quad \frac{\ddot{Z} \mathrm{r} 2,00}{100,30}
$$

\section{Zoisit vom Fichtelgehir e (Gefrees).}

\section{Kieselsäure \\ Thonerde \\ Eisenoxyd \\ Manganoxydul \\ Kalkerde \\ Glüherlust}

\section{Zoisit (?) non Falligl, Tyrol.}

Kohlensäure

Kieselsäure

Thonerde

Eisenoxyd

Eisenoxydul

Manganoxydul

Kalkerde

Talkerde

Glühverlust

\begin{tabular}{cc} 
Bucholz. & Geffkeu. \\
40,25 & 40,03 \\
30,25 & 29,83 \\
4,50 & 4,24 \\
$-5,50$ & 7,55 \\
22,50 & 18,85 \\
2,00 & - \\
\hline 99,50 & 100,50
\end{tabular}

a. Geffken.

$\widehat{40,74}$

28.94

5,19

1,78

20,32

$\frac{4,75}{-}$ b.

Hermann.

1,13

40,95

30,34

$\overline{4,96}$

$\overline{21,56}$

$\overline{0,56}$

4. Sogenannter Mejomit von Stersing in Tyrol.

(Von Weiss zuerst zum Epidot gestellt.)

Kieselsäure
Thonerde
Eisenoxyd
Manganoxydul
Kalkerde
Natron, Kali
Wasser

Kieselsäure

Thonerde

Manganoxydul

Kalkerdo

Wasser a. b.

$\begin{array}{cc}\text { a. } & \text { b. } \\ \text { Stromeyer. } & \text { Richter. }{ }^{* *} \\ \mathbf{3 9 , 9 1} & \mathbf{4 0 , 5 7} \\ \mathbf{3 1 , 9 7} & \mathbf{3 2 , 6 7} \\ \mathbf{2 , 4 4} & \mathbf{5 , 1 1} \\ 0,17 & - \\ \mathbf{2 3 , 8 5} & \mathbf{2 0 , 8 2} \\ \mathbf{0 , 8 9} & - \\ 0,95 & 1,22 \\ 100,18 & \mathbf{1 0 1 , 3 9}\end{array}$

*) Sitzungsber. d. Wien. $\Lambda k$. d. Wiss. J. f. prakt. Chem. LXIV 31 b.

*) Haidinger Ber. üb. d. Mitth. v. Fr. d. Naturw. 3, 114. 
Um die Frage zu entscheiden, ob der Zoisit dieselbe Zusammensetzung wie der Epidot habe, was bisher zwal angenommen wurde, jedoch durch die angeführten Ana lysen nicht scharf bewiesen ist, hielt ich es für angemes. sen, die ausgezeichnetsten Vorkommnisse des Minerals von neuem $z u$ untersuchen. Den Stoff $z u$ den Analysen verdanke ich dem $K$. Mineralien-Cabinet, aus welchem Herr G. Rose ibn mir übergab, so wie der gefälligen Mittheilung des verstorbenen Custos P. Partseh in Wien und des Herrn Dr. Krant $z$ in Bonn.

Mit besonderer Sorgfalt wurde das specifische Gewicht bestimmt.

Von jeder Abänderung wurden mindestens zwei Analysen gemacht, indem nämlich eine Probe durch Schmelzen mit kohlensaurem Natron aufgeschlossen wurde. während eine andere nach vorgängigem Glühen durch Chlorwasserstoffsäure sich leicht zersetzen liess, und eine vollkommene Gallerte hildete.

Beim Erhitzen erleidet der Zoisit stets einen Gewichtsverlust. Derselbe tritt schon bei mässigem Rothglühen über der Lampe ein und liesse sich wahrscheinlich durch längere Dauer der Operation auf diese Art bis zur vollständigen Austreibung aller flüchtigen Stoffe bringen; schneller gelangt man freilich zum Ziel, wenn man das Mineral einige Minuten der Wirkung eines Gasgebläses aussetzt. Die kleinen Bruchstücke, welche zu diesen Versuchen dienten, hatten dann stets ihre Durchscheinheit verloren; sie waren opak, rissig, öfter bräunlich gefärbt, doch fand nie, selbst bei Anwendung von pulvrigem Material, ein wirkliches Sintern oder gar Schmelzen statt.

Der Gewichtsverlust, welchen der Zoisit auf diese Weise erleidet, beträgt $2-2^{2}$ p. C. Je frischer, durchscheinender und härter er ist, um so geringer ist dieserVerlust. Diejenigen Abänderungen, bei denen er 3 p. C. übersteigt, z. B. aus der Fusch und dem Meiggerthal, zeigen schon durch ihre äussere Beschaffenheit auf einen nicht mehr ganz frischen Zustand der Masse hin, denn sie sind weicher, minder durchscheinend, und es lösen sich

Journ. f. prakt. Chemie. LXX, 4. 
von der Spaltungsfläche sehr dünne glimmerähnliche Blättchen ab.

Bei den Versuchen, die Natur der flüchtigen Stoffe zu ermitteln, wurde das grobgepulverte Mineral auf einem Platinblech in eine etwa 2 lange Platinröhre gebracht, und konnte darin über einer Gaslampe, wie sie zur Elementaranalyse dient, hinreichend stark erhitzt werden. Es wurde trockne kohlensäurefreie atmosphärische Luft darüber geleitet, welche dann eine Chlorcalciumröhre und Barytwasser passirte. Es entwickelte sich neutral reagirendes Wasser und ein wenig Kohlensäure, wiewohl ich nicht ganz so viel derselben erhielt, als dem Gewichtsverlust des Minerals entsprach.

Die Quantität des Wassers, auch wenn der volle Verlust als solches genommen wird, ist allerdings so gross, dass es als Hydratwasser betrachtet werden könnte. Allein seine Menge ist in den einzelnen Abänderungen nicht gleich, und rührt sammt der begleitenden Spur Kohlensäure otrenbar von dem Angriff atmosphärischer Gewässer auf das Mineral her, dem auch die frischeren Varietäten ausgesetzt waren. Ich glaube daher, dass der Zoisit gleich dem Epidot ursprünglich wasserfrei ist.

Im Folgenden ist a die Analyse mit kohlensaurem Natron; $b$ dieselbe auf wasserfreie Substanz berechnet; c die Analyse des geglühten Minerals mit Chlorwasserstoffsäure; $d$ das Mittel von $b$ und $c$.

\section{Zoisit von der Saualpe.}

Spec. Gew. $=3,353$.

Die Begleiter waren Quarz und Granat.

\begin{tabular}{lrrrr} 
& $a$. & \multicolumn{1}{c}{ b. } & \multicolumn{1}{c}{$c$} & d. \\
Kieselsäure & $\mathbf{4 0 , 0 8}$ & $\mathbf{4 1 , 1 5}$ & $\mathbf{4 1 , 8 7}$ & $\mathbf{4 1 , 5 1}$ \\
Thonerde & $\mathbf{2 8 , 7 0}$ & $\mathbf{2 9 , 4 7}$ & $\mathbf{2 8 , 3 2}$ & $\mathbf{2 8 , 9 0}$ \\
Eisenoxyd & $\mathbf{3 , 5 0}$ & $\mathbf{3 , 6 0}$ & $\mathbf{4 , 3 7}$ & $\mathbf{3 , 9 8}$ \\
Kalkerde & $\mathbf{2 4 , 2 7}$ & $\mathbf{2 4 , 9 2}$ & $\mathbf{2 4 , 6 4}$ & $\mathbf{2 4 , 7 8}$ \\
Talkerde & 0,84 & $\mathbf{0 , 8 6}$ & $\mathbf{0 , 3 0}$ & $\mathbf{0 , 5 8}$ \\
Glühverlust & $\mathbf{2 , 0 9}$ & $\mathbf{1 0 0 , 0 0}$ & $\mathbf{9 9 , 5 0}$ & $\mathbf{9 9 , 7 5}$ \\
& 99,48 & & &
\end{tabular}

Es ist bemcrkenswerth, dass von den Früheren Thomson allein die Menge der Kieselsäure und des Kalks richtig gefunden hat. 


\section{Zoisit vom Fichtelgebinge (Gefrees).}

Spec. Gew. $=3,361$.

Ein bekanntes Vorkommen.

\begin{tabular}{|c|c|c|c|c|}
\hline & a. & b. & $\left.c^{*}\right)$ & d. \\
\hline Kieselsäure & 40,21 & 11,07 & 11,30 & 41,18 \\
\hline Thonerde & 29,00 & 29,62 & 31,19 & 30,40 \\
\hline Eisenoxyd & 2,51 & 2,50 & 3,10 & 2,83 \\
\hline Kalkerde & 24,31 & $2,4,82$ & 24,93 & 24,87 \\
\hline \multirow{3}{*}{$\begin{array}{l}\text { Talkerde } \\
\text { Glühverlust }\end{array}$} & 0,26 & 0,27 & 0,23 & 0,25 \\
\hline & 2,08 & 98,34 & 100,75 & 99,53 \\
\hline & $98,3 \overline{7}$ & & & \\
\hline
\end{tabular}

III. Zoisit von Goshen, Massachusets.

Spec. Gew. $=3,341$; nach dem Glühen $\cdots 2,726$.

Hleicht dem vorigen ganz und gar.

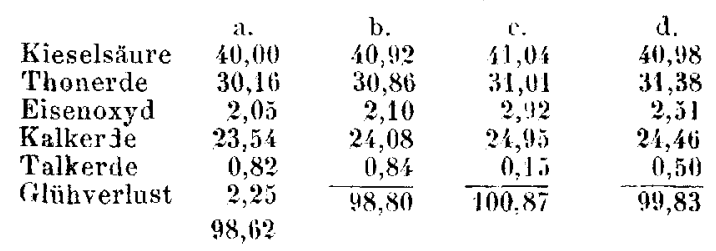

IV. Zoisit von stersing, Tyrol.

Spec. Gew. $=3,352$.

Glühverlust $=\mathbf{2 , 0 4} \mathrm{p} . \mathrm{C}$.

Ich habe diese Abänderung nur im geglühten Zustande untersucht.

$\begin{array}{lr}\text { Kieselsäure } & \mathbf{1 0 , 8 2} \\ \text { Thonerde } & \mathbf{3 0 , 9 7} \\ \text { Fisenoxyd } & \mathbf{2 , 1 1} \\ \text { Kalkerde } & \mathbf{2 4 , 6 5} \\ \text { Talkerde } & \mathbf{0 , 2 4} \\ & \mathbf{9 8 , 7 9}\end{array}$

V. Zoisit aus dem Fuschthal, Pinzgau.

Spec. Gew. $=3,251$.

Dünnstänglige Massen, in Quarz eingewachsen, gelblich-grau, sehr zerbrechlich, weicher als die früheren, hie und $d a$, besonders auf den Spaltungstlächen, mit silberweissen glimmerähnlichen Schuppen bedeckt.

*) Vittel yon zwei Analysen. 


\begin{tabular}{lrrrr} 
& a. & b. & c. & \multicolumn{1}{c}{ d. } \\
Kieselsäure & $\mathbf{4 1 , 4 4}$ & $\mathbf{4 3 , 0 2}$ & $\mathbf{4 4 , 0 2}$ & $\mathbf{4 3 , 5 2}$ \\
Thonerde & $\mathbf{2 7 , 1 5}$ & $\mathbf{2 8 , 1 9}$ & $\mathbf{3 0 , 8 6}$ & $\mathbf{2 8 , 1 9}$ \\
Eisenoxyd & $\mathbf{2 , 9 4}$ & $\mathbf{3 , 0 5}$ & $\mathbf{3 , 0 5}$ \\
Kalkerde & $\mathbf{2 2 , 8 1}$ & $\mathbf{2 3 , 6 8}$ & $\mathbf{2 3 , 5 1}$ & $\mathbf{2 3 , 6 0}$ \\
Talkerde & $\mathbf{1 , 2 3}$ & 1,28 & $\mathbf{1 , 2 4}$ & $\mathbf{1 , 2 6}$ \\
Glühverlust & $\mathbf{3 , 6 7}$ & $\mathbf{9 9 , 2 2}$ & $\mathbf{9 9 , 6 3}$ & $\mathbf{9 9 , 6 2}$
\end{tabular}

VI. Zisit von Meiggerthal (Saasthal) am Monte Rosa. Spec. Gew. $=3,280$.

Diese Abänderung zeichnet sich durch eine schöne grïne Farbe aus, welche an den Amazonenstein erinnert. Die stängligen Aggregate sind mit Quarz verwachsen, lassen sich leicht zerbrechen und sind an manchen Stellen mit grünen Glimmer oder Chlorit ähnlichen Blättchen überzogen.

\begin{tabular}{|c|c|c|c|c|}
\hline & a. & b. & c. & d. \\
\hline Kieselsāure & 41,80 & 13,17 & 44,32 & 43,74 \\
\hline Thonerde & 28,62 & 29,46 & 29,00 & 20,23 \\
\hline Eisenoxyd & $2 ; 82$ & 2,90 & 3,45 & 3,18 \\
\hline Kalkerdc & 21,34 & 22,04 & 22,58 & 22,31 \\
\hline Talkerde & 0,66 & 0,68 & 0,34 & 0.59 \\
\hline Kali & - & - & 0,93 & 0,93 \\
\hline \multirow[t]{2}{*}{ Gluhverlust } & 3,18 & 98,25 & $\overline{100, \overline{82}}$ & 99,98 \\
\hline & 98,42 & & & \\
\hline
\end{tabular}

Sauerstoffgehalt in $\mathrm{d}$ :

\begin{tabular}{|c|c|c|c|c|c|c|}
\hline & I. & II. & III. & IV. & V. & $V 1$. \\
\hline & $21, \check{a} \tilde{3}$ & 21,38 & $21,2 \bar{i}$ & 21, & 22,59 & 22,71 \\
\hline & 13,49 & 14,19 & 14,65 & 14,46 & 13,16 & 13,65 \\
\hline & 1,14 & 0,85 & $0,7 \tilde{a}$ & 0,0 & 0,91 & $0,9 \mathrm{j}$ \\
\hline & ) & 10 & 6,99 & 7,04 & $8 ?$ & 6,37 \\
\hline & 23 & 10 &, 20 & 0,10 & 0,50 & $0,3 y$ \\
\hline
\end{tabular}

Es ist demnach das Verhältniss des Sauerstoffs: $\dot{\mathrm{R}}: \quad \dddot{R}: \ddot{\mathrm{S}} \mathrm{i}$

I. $7,31: 14,68: 21, \tilde{\jmath} 5=1: 2,00: 2,95$

II. $7,20: 15,04: 21,38:=1: 2,09: 2,97$

III. $7,19: 15,40: 21,27=1: 2,14: 2,96$

IV. $7,14: 15,09: 21,19=1: 2,11: 2,97$

V. $7,24: 14,07: 22,59=1: 1,94: 3,12$

VI. $6,76: 14,60: 22,71=1: 2,16: 3,36$ 
Es kann hiernach nicht länger zweifelhaft sein, dass das Sauerstoffverhältniss $=1: 2: 3$, der Zoisit mithin dem Epidot gleich zusammengesetzit sei,

$$
\dot{\mathrm{Ca}}_{3} \mathrm{Si}+2 \mathrm{~A} \mathrm{Asi} \text {. }
$$

Da nun auch beim Mejonit dasselbe Sauerstoffverhältniss obwaltet, so wäre die Verbindung.

$$
\dot{\mathrm{R}}_{3} \dddot{\mathrm{S}} \mathrm{i}+2 \dddot{\mathrm{R}} \ddot{\mathrm{S}} \mathrm{i}
$$

di- oder trimorph.

Die vorstehenden Untersuchungen geben mir Anlass, einige Bemerkungen über die Zusammensetzung des Epidots zu machen, welche in neuerer Zeit Gegenstand der Arbeiten von Hermann, Stockar-Escher, Scheerer, von mir u. A. gewesen ist. Die Anzahl der vorhandenen Analysen ist mithin gross genug, um ein sicheres Urtheil über die chemische Zusammensetzung des Minerals zu gestatten.

Dem Zoisit qualitativ gleich, unterscheidet sich der Epidot vornämlich durch einen grösseren Eisengehalt, welcher als Oxyd von 9-16 p. C. steigt, womit eine $A b-$ nahme der Thonerde (bis 20 p. C.) verbunden ist.

Schon aus den älteren Analysen wurde klar, dass das Verhältniss 'des Sauerstoffs der Kalkerde, der Thonerde (und des Eisenoxyds) und der Kieselsäure $=1: 2: 3$ sei, dass der $\mathrm{E}$. mithin aus Drittelsilikaten bestehe.

Ich habe die zuverlässigen Analysen von Epidot berechnet, welche wir jetzt besitzen. Sie betreffen den $E$. von Arendal, Bourg d'Oisans, Traversella, vom Haslithal, Sustenhorn, Magis, Kaverdiras, St. Gotthardt, so wie die russischen Vorkommen von Achmatowsk, der Schumnaja, Burowa und Werchneiwinsk, und erreichen die Anzahl von 28. Wird der Sauerstoff des Kalks (wozu bisweilen ein wenig Talkerde, selbst Alkali, kommt) $=1$ gesetzt, so ist der Sauerstof $\mathrm{f}$ von Thonerde und Eisenoxyd, 1,8 - 2,6 als Extreme; in 4 Analysen ist er genau $=2$, in 20 steigt er höchstens auf 2,3. Der Sauerstoff der Kieselsäure liegt zwischen 2,7 und 3,1 , beträgt in 8 Fällen genau 3 , bleibt aber in der Mehrzahl etwas darunter; das Mittel sämmtlicher Analysen ist die Proportion 1:2,2:2,9, wofür als 
nächst einfache $1: 2: 3$ genommen werden muss, die alte Formel des Epidots mithin sich bestätigt.

Während nun im Zoisit der Fisengehalt so gering ist (als Oxyd 2-31/2 p.C.) dass auf 1 At. Fisenoxyd 12-24 At. Thonerde kommen, ist dies Verhältniss beim

E. von Lole (Magis) $=1: 6$, vom Sustenhorn, St. Gotthardt, Kaverdiras $=1: 5$, von Guttannen (Haslithal) $=\mathbf{1}: \mathbf{4}$, von Arendal, Traversella, Bourg d'Oisans, Achmatowsk, Werchneiswinsk und der Schumnaja $=$ $1: 2$, von Burowa $=1: 1^{1 / 2}$

Es ist bemerkenswerth, dass der Sauerstoff der Sesquioxyde in der grossen Mehrzahl der Analysen immer etwas mehr als das Doppelte von dem der Monoxyde (im Mittel 2,2) beträgt. Der Grund davon kann in einem Gehalt an Eisenoxydul liegen. Auch hat Hermann dasselbe in allen Epidoten gefunden, und zwar in Mengen von 2-51/2 p. C.

Gewiss ist es sehr schwer, wo nicht unmöglich, in derartigen Verbindungen beide Oxyde des Eisens genau zu bestimmen, und die Methode Hermann's bürgt durchaus nicht für die Richtigkeit der Resultate. Zwei Epidotvarietäten von Arendal gaben $\mathrm{ihm} 1,86$ und $\mathbf{5 , 2}$ p. C. Eisenoxydul; eine von Bourg d'Oisans $\mathbf{5 , 5 5}$ p. C. Nun habe ich beide Epidote bereits vor längerer Zeit auf Eisenoxydul geprüft, und dasselbe that S tockar-Escher in Betreff der schweizer Varietäten. Wir fanden jedoch kein oder nur geringe Spuren von Eisenoxydul. Ganz kürzlich ist es mir indessen gelungen, durch volumetrische Anwendung von übermangansaurem Kali in dem E. von Arendal und dem ron Guttannen im Haslithal 1,65 - 1,95 p. C. Eisenoxydul zu bestimmen, obwohl ich glaube, dass diese Angabe zu hoch ist.

Wie wenig Einfluss jedoch das Eisenoxydul auf dic einfache Sauerstoffproportion 1:2:3 hat, ergieht eine Berechnung der Epidote, bei welchen jenes Oxyd bestimmt worden ist. Man erhält nämlich dann das Verhältniss = $1,1: 2,1: 3$. 
Scheerer berechnet aus seinen eigenen und StockarEscher's Analysen das mittlere Verhältniss $=1: 21 / 4: 3$ $=4: 9: 12$, und verwirft mithin die bisherige und von mir vertheidigte Formel des Epidots. Diese Zahlen drücken aber auch das oben angeführte Mittel aller Analysen aus, da $1: 2,2: 2,9=103: 228: 300$, statt dessen $\mathrm{Sch}$ eerer $100: 225: 300$ setzt. Scheerer hat sich also genau an die Resultate der Analysen gehalten.

Es ist nothwendig, bei dieser Gelegenheit darauf aufmerksam zu machen, dass in den letzten Jahren mehrfach die analytischen Resultate von Mineralien nach anderen Grundsätzen als den bisher befolgten, gedeutet zu werden pflegen.

Berzelius hatte gelehrt, dass das Rusultat der Analyse einfachen Aequivalentverhältnissen entsprechen müsse, und dass, da die Schärfe der Methoden, die Reinheit und Frische des Materials bei Mineralien immer nur eine $\Lambda \mathrm{n}$ näherung gestatten, das nächste einfache Verhältniss zu wählen sei.

Diesem Princip entgegen findet man jetzt nicht selten die Neigung, ein complicirteres Verhältniss zu wählen, wenn dasselbe sich dem empirischen Resultat besser anpasst. Dadurch treten neue Formeln an die Stelle der einfachen ältern, und das Gebiet der natürlichen Silicate wird zum Schauplatz einer Unzahl von Versuchen.

Bei der höchsten Achtung vor dem Werth der Thatsachen wird man doch nicht umhin können, die Umstände $z u$ berücksichtigen, welche die $\Lambda$ nalyse der natürlichen Silicate schwierig machen, und bei der Deutung des Resultats wohl erwogen werden müssen. Hält man sich streng an dieses, auch wenn es minder einfach ausfällt, so vindicirt man der Analyse und ihren Operationen eine sehr grosse Schärfe, insbesondere betrachtet man die Substanz als rein und ursprünglich. Das Urtheil über jene steht nur dem Analytiker zu; eigene Erfahrung hat mich allerdings gelehrt, das Vertrauen auf diesen Punkt nicht allzuhoch in Anschlag zu bringen. Was aber die Reinheit und Ursprünglichkeit betrifft, in welcher Silicate vorkommen, 
so haben gerade die neueren Arbeiten auf diesem Felde gelehrt, wie selten sie anzutreffen seien.

Der Zoisit und der Epidot enthalten einige Procent. Wasser. Sind sie ursprünglich Hydrate, oder ist das Wasser erst später hinzugekommen, ist die Hydratbildung mit einer chemischen Veränderung des Minerals verknüpft? Schon vor Iängerer Zeit theilte ich mit, dass der E. von Arendal beim Glühen $2 \mathrm{p}$. C. verliert. Scheerer und Stockar-Escher bestimmten das Wasser neuerlich und fanden es in allen Epidoten $\mathbf{2 - 2 , 4}$ p. C. hetragend. Auch die Zoisite verlieren, wie ich gefunden und oben mitgetheilt habe, 2-32/s p. C. Wasser beim G]ühen*), und zwar am meisten diejenigen, welche durch ihr Ansehen schon auf eine anfangende Zersetzung schliessen lassen, deren spec. Gewicht etwas unter dem der übrigen liegt, und welche das Minimum von Kalk, das Maximum von Talkerde enthalten. Der $\mathbf{E}$. ist oft mit Kalkspath verwachsen, dessen Bildung von einem Angriff kohlensaurer Wässer auf Epidot und andere primitive kalkhaltige Silicate herrühren kann. Wird es nun befremden können, dass die Mittelzahl der Epidot-Analysen für den Kalk und auch für die Kieselsäure kleinere Werthe giebt, als dem Sauersfoffverhältniss $1: 2: 3$ gemäss ist? Dass das Mincral einen Schritt zur Hydratbildung gethan hat?

Der Fortschritt der Wissenschaft, die Vermehrung der empirischen Irülfsmittel, zur Kenntniss der inneren und äusseren Natur der Körper zu gelangen, rufen neue Richtungen hervor, in denen die Forschung sich bewegt. So ist es jetzt in der Mineralogie der Fall. Möchten aber doch Alle sich daran erinnern, dass allein die Facta bleibenden Werth besitzen, unsere Hypothesen dagegen um so vergänglicher sind, je weniger sie auf einfache Verhältnisse Rücksicht nehmen.

*) Schon Buchol\% giebt für den Z. vom Fichtelgebirge? p. C. Glöhverlust an. 